\title{
Factors Associated with Adherence to Colorectal Cancer Screening among Moderate Risk Individuals in Iran
}

\author{
Zahra Taheri-Kharameh ${ }^{1}$, Farsad Noorizadeh $^{2}$, Samira Sangy $^{2}$, Hadi Zamanian$^{2 *}$, \\ Ali Reza Shouri-Bidgoli ${ }^{1}$, Helaleh Oveisi ${ }^{3}$
}

\begin{abstract}
Background: Colorectal cancer is one of the most common neoplasms in Iran. Secondary prevention (colorectal cancer screening) is important and a most valuable method of early diagnosis of this cancer. The objectives of this study were to determine the factors associated with colorectal cancer screening adherence among Iranians 50 years and older using the Health Belief Model. Materials and Methods: This cross-sectional study was conducted from June 2012 to May 2013. A convenience sample of 200 individuals aged 50 and older was recruited from the population at outpatient clinics in teaching hospitals. Data gathering tools were the Champions health belief model scale (CHBMS) with coverage of socio-demographic background and CRC screening information. Multiple logistic regression was performed to identify factors associated with colorectal cancer screening adherence. Results: The mean age of participants was $62.5 \pm 10.8$ and $75.5 \%$ were women. A high percentage of the participants had not heard or read about colorectal cancer $(86.5 \%)$ and CRC screening $(\mathbf{9 3 . 5 \%})$. Perceived susceptibility to colorectal cancer had the lowest percentage of all of the subscales. Participants who perceived more susceptibility $(\mathrm{OR}=2.99$; CI 95\%: 1.23-5.45) and reported higher knowledge $(\mathrm{OR}=1.29$; CI 95\%: 1.86-3.40) and those who reported fewer barriers (OR =.37; CI 95\%:.21- .89), were more likely to have carried out colorectal cancer screening. Conclusions: Our findings indicated that CRC knowledge, perceived susceptibility and barriers were significant predictors of colorectal cancer screening adherence. Strategies to increase knowledge and overcome barriers in risk individuals appear necessary. Education programs should be promoted to overcome knowledge deficiency and negative perceptions in elderly Iranians.
\end{abstract}

Keywords: Colorectal cancer screening - adherence - knowledge - health belief model - Iran

Asian Pac J Cancer Prev, 16 (18), 8371-8375

\section{Introduction}

Colorectal cancer (CRC) is third most common cancer diagnosed in males and the second in females throughout the developed world (Haggar and Boushey 2009). It has been shown that the incidence of CRC is increasing in Asia (Sung et al., 2005). In Iran, gastrointestinal cancers among males and breast cancer among females are the most prevalent cases (Alireza et al., 2005). Early detection through screening is the best way to reduce mortality of CRC (Katz et al., 2007). Regular screening in those 50 years of age and older is helpful to decrease the one third of CRC deaths (Greiner et al., 2005). Despite the impact of screening on timely and treatable stage of the cancer, screening rates are low (Henley et al., 2010). The lack of participation in screening programs can be described based on health belief model (HBM) (Taylor et al., 1999). It is one of the most widely recognized and used models in health behavior to find why people did not participate in health-screening programs. If attitudes related to health behavior can be identified, health protection interventions for attitude change can be developed, and an increase in desirable health behavior would result (Champion 1984). The HBM is composed of six concepts: 1) perceived susceptibility (a person's opinion regarding the chances of developing a condition). 2) Perceived Severity (one's belief regarding the seriousness of a condition), 3) barriers (one's beliefs regarding the total barrier costs of implementing the recommended action), 4) benefits (the opinion of the effectiveness of actions to reduce the risks of a condition), 5) health motivation related to performing the health behaviors, 6) self efficacy (one's beliefs about their capabilities to take the recommended action) (Ueland et al., 2006). The HBM-based research studies have determined these components of HBM as correlates of cancer prevention and screening behaviors (Janz et al., 2003; Palmer et al., 2007; McFarland 2013; Ebu et al., 2015). 
Various studies have sought to identify psychosocial factors associated with participation in CRC screening (Gili et al., 2006; Cole et al., 2011; Gonzalez et al., 2012; Jorgensen et al., 2013). But few studies have examined beliefs and behaviours related to CRC screening in Iranian population. A previous study about beliefs associated with CRC screening in an Iranian adult population based on preventive health model, showed that subjects mostly reported poor attitude about CRC screening (Salimzadeh et al., 2011). Javadzadeh et al found a significant relationships between knowledge and all domains of HBM excepting perceived benefits among individuals more than 50 years old (Javadzade et al., 2012).

It is necessary to identify the factors associated with colorectal cancer screening adherence to increase this adherence (Gimeno García, 2011). As well, since adherence to CRC screening could be influenced by culture (Wang et al., 2006), identifying these factors in different cultures is useful to better understanding of people's beliefs which assist us in developing ways to increase use of CRC screening procedure (Brennenstuhl et al., ). Also effective cancer screening programs depends on accurate assessing patients' needs and designing helpful educational strategies. The purpose of this study was to determine the predictive factors of CRC screening adherence among Iranians aged 50 and older based on the HBM.

\section{Materials and Methods}

\section{Design and participants}

This cross sectional study has been performed in June 2012 to May 2013 in Qom City, a provincial city in the central region of Iran. A convenience sample of 200 individuals aged 50 and older was recruited from population at outpatient clinics in three teaching hospitals. The inclusion criteria were being 50 years or older, not having had CRC and ability to communicate in Persian language. Verbal informed consent was obtained from the participants after the purpose of the study was explained to them. The questionnaire's items were read for those individuals who were unable to read it and their answers were checked by the researcher. Questionnaire completion took 10 to 15 minutes. Questionnaires were collected and revised by the researcher.

\section{Questionnaires:}

We used two instruments as follows: A questionnaire was used to collect socio-demographic background and CRC screening information. The questionnaire consisted of questions about age, gender, marital status, educational status, employment, and economic status, Residence, family history of CRC, knowledge about the disease and screening for CRC and screening status.

Champion's Health Belief Model Scale (CHBMS), a 36-item questionnaire, was used to assess health beliefs and perceptions about screening according to six subscales; Perceived Susceptibility (5 items), Perceived Seriousness (7 items), Perceived Benefits (6 items), Perceived Barriers (6 items), health motivation (7 items) and Confidence (5 items). Items were formatted with a 5-point Likert scale, from 1 (strongly disagree) to 5 (strongly agree). This Scale was originally developed and revised by Champion (Champion 1999). Previous evaluation of the original as well as the Persian version of CHBMS indicated good reliability and validity (Jacobs 2002; Kharameh et al., 2013).

CRC screening status was assigned as the dependent variable and was determined based on the self reports of study participants. The individuals were considered adherent to CRC screening if they had undergone at least one screening within the recommended 5-year screening interval and expected to undergo an additional screening in the future. The individuals considered non-adherents if they did not meet these criteria.

\section{Statistical analysis}

Data analysis was done in SPSS version 16.00 for Windows. Descriptive analysis such as frequency, mean and standard deviation was used to describe socio-demographic data and components of HBM in participants. Chi-squared analysis of variance was used for comparison. The level of significance considered less than 0.05 . Multiple logistic regression was used to examine the associations of the socio-demographic characteristics, knowledge and HBM components with CRC screening adherence. The independent variables were entered for logistic regression analysis as a group, levels of significance and other parameters such as $2 \log$ likelihood and wald were examined, and the best fit model was systematically determined by eliminating non significant variables. Then selected variables were entered to new. Iterations step by step. The factors that were found to be more significant in the previous models were included in the next multiple logistic regression model which led to a final model that well predicted adherence to screening.

\section{Ethical considerations}

All ethical issues were considered. Participation in this study was voluntary and all participants were included after informed consent. Formal approvals to conduct the project and collect the data were obtained from the ethics committees of Qom University and the study sites.

\section{Results}

\section{Sample characteristics}

Mean age of participants was $62.47(\mathrm{SD}=10.78)$ and 75.5 percent of participants were male. Most of the patients $(88 \%)$ were from urban districts. most of them were Iranian (96.5\%) and with Persian ethnicity (62.8\%). 76.3 percent of patients were married. The majority of the sample (89\%) has no family history of CRC. (Table 1)

\section{Knowledge of CRC}

A high percentage of the participants (86.5\%) never hear or read about CRC, whereas $13.5 \%$ of the participants only had read or heard about CRC. The source of their information was mostly family members $(30 \%)$, Physician and nurse $(23 \%)$, Friends $(16.7 \%)$, and television and radio $(13.3 \%)$, Newspapers and magazines $(13.3 \%)$ and others $(3.3 \%)$. 


\section{Knowledge of CRC screening}

A high percentage of the participants $(93.5 \%)$ had no knowledge about CRC screening and $6.5 \%$ read or heard some information about CRC screening. The information sources the included family members $(38.9 \%)$, television and radio (22.2\%), Newspapers and magazines (13.3\%), Physician and nurse (11.1\%) and others (3.3\%).

\section{Perceptions and beliefs About CRC Screening}

Table 2 shows the means, and standard deviations of

Table 1. Sample Characteristics $(N=200)$

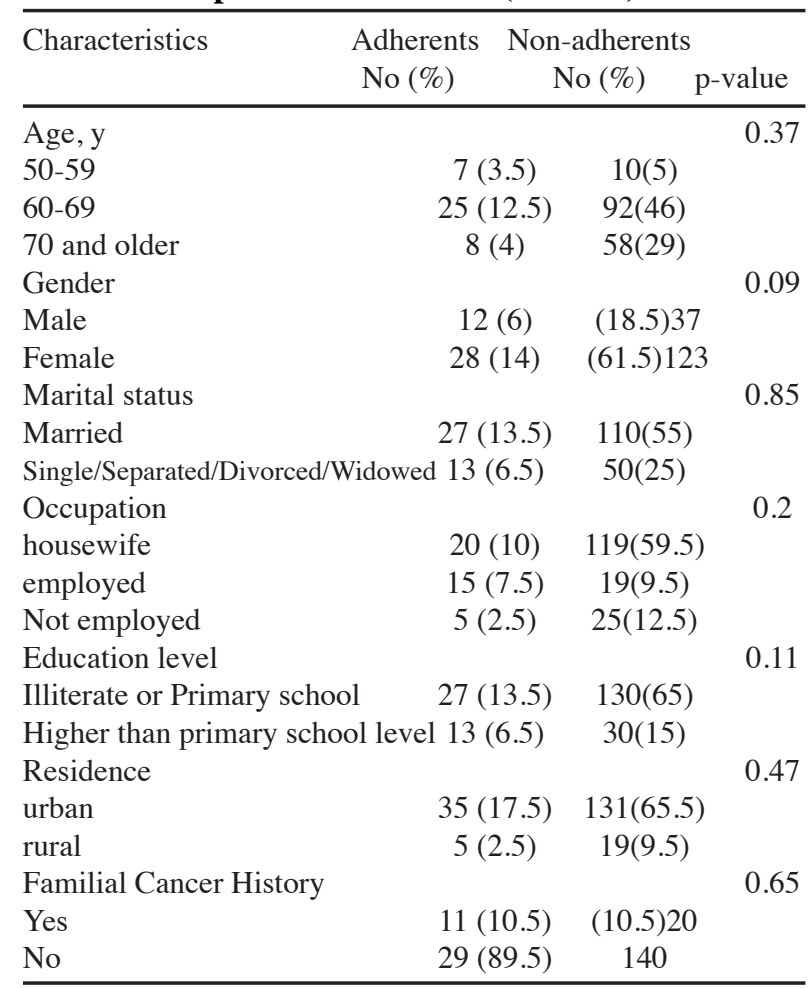

the HBM variables. Participants rated seriousness and susceptibility as lower than average indicating they did not perceive colorectal cancer as a serious disease or that it would affect them. Benefits, barriers, health motivation and Confidence were rated above average. HBM Subscales was classified positive, neutral, and negative perception. This study clarified that the participants had positive perceptions toward each of the subscales as follows: $8 \%$ susceptibility, $66 \%$ seriousness, $88 \%$ benefits, $25 \%$ barriers, $68.5 \%$ health motivation and $78 \%$ Confidence; negative perception: $60 \%$ for susceptibility, $32.5 \%$ for seriousness, $10 \%$ for benefits, $25 \%$ for barriers, $19 \%$ for health motivation and $16 \%$ Confidence (Table 3 ).

Table 2. Mean and Standard Deviation Values of Health Beliefs

\begin{tabular}{lcccc}
\hline Scale & $\begin{array}{c}\text { Number of } \\
\text { Scale Items }\end{array}$ & $\begin{array}{c}\text { Possible } \\
\text { score range }\end{array}$ & $\begin{array}{c}\text { Mean } \\
\text { Score }\end{array}$ & $\begin{array}{c}\text { Standard } \\
\text { Deviation }\end{array}$ \\
\hline Susceptibility & 5 & $5-25$ & 2.42 & 0.89 \\
Seriousness & 7 & $7-35$ & 3.22 & 0.74 \\
Benefits & 6 & $6-30$ & 3.79 & 0.65 \\
Barriers & 6 & $6-30$ & 3.36 & 0.81 \\
Health motivation & 7 & $7-35$ & 3.51 & 0.67 \\
Confidence & 5 & $5-25$ & 3.43 & 0.54 \\
\hline
\end{tabular}

Table 3. Subscales of HBM Classification

\begin{tabular}{lclc}
\hline Scale & Negative & Neutral & Positive \\
\hline Susceptibility & $120(60)$ & $64(32)$ & $16(8)$ \\
Seriousness & $65(32.5)$ & $13(6.5)$ & $122(61)$ \\
Benefits & $20(10)$ & $14(7)$ & $166(83)$ \\
Barriers & $126(63)$ & $24(12)$ & $50(25)$ \\
Health motivation & $38(19)$ & $25(0.25)$ & $137(68.5)$ \\
Confidence & $32(16)$ & $11(5.5)$ & $156(78)$ \\
\hline
\end{tabular}

Table 4. First Logistic Regression Model of Predicting Factors of CRC Screening Adherence

\begin{tabular}{|c|c|c|c|c|c|c|c|}
\hline \multirow[t]{2}{*}{ Variable } & \multirow[t]{2}{*}{$\mathrm{B}$} & \multirow[t]{2}{*}{ S.E. } & \multirow[t]{2}{*}{ Wald } & \multirow[t]{2}{*}{ Sig. } & \multirow[t]{2}{*}{$\operatorname{Exp}(B)$} & \multicolumn{2}{|c|}{ Interval confidence $\% 95 \mathrm{CI}$} \\
\hline & & & & & & Lower & Upper \\
\hline Age & 0.014 & 0.02 & 0.35 & 0.55 & 1.01 & 0.97 & 1.05 \\
\hline Gender Male1.0 (ref) & 1.25 & 0.62 & 4.07 & 0.04 & 3.52 & 1.03 & 11.94 \\
\hline Education & 0.075 & 0.18 & 0.15 & 0.69 & 1.07 & 0.74 & 1.558 \\
\hline Marital status & -0.04 & 0.18 & 0.06 & 0.8 & 0.955 & 0.66 & 1.367 \\
\hline Occupation & -0.12 & 0.19 & 0.43 & 0.51 & 0.881 & 0.6 & 1.28 \\
\hline Familial Cancer History & 0.12 & 0.54 & 0.05 & 0.82 & 1.13 & 0.38 & 3.29 \\
\hline CRC knowledge & 0.33 & 0.63 & 0.28 & 0.29 & 1.4 & 0.4 & 4.89 \\
\hline Susceptibility & 1.83 & 1.13 & 2.62 & 0.1 & 6.23 & 0.68 & 7.06 \\
\hline Seriousness & -1.53 & 0.91 & 2.81 & 0.09 & 0.21 & 0.036 & 1.29 \\
\hline Benefits & 0.64 & 0.35 & 3.27 & 0.07 & 1.89 & 0.94 & 3.79 \\
\hline Barriers & 0.414 & 0.47 & 0.76 & 0.18 & 1.51 & 0.59 & 3.82 \\
\hline Health motivation & -0.83 & 0.57 & 2.16 & 0.14 & 0.43 & 0.14 & 1.32 \\
\hline Confidence & -0.83 & 0.57 & 2.16 & 0.14 & 0.43 & 0.14 & 1.32 \\
\hline
\end{tabular}

Table 5. Final Logistic Model of Predicting Factors of CRC Screening Adherence

\begin{tabular}{lccccccc}
\hline Variable & B & S.E. & Wald & Sig. & Exp(B) & \multicolumn{2}{c}{ Interval confidence \%95 CI } \\
\cline { 5 - 7 } & & & & & Lower & Upper \\
\hline CRC Knowledge & 2.19 & 1.01 & 4.7 & 0.03 & 2.99 & 1.23 \\
Susceptibility & 1.45 & 0.82 & 3.15 & 0.04 & 1.29 & 1.86 \\
Barriers & -0.976 & 0.364 & 6.18 & 0.01 & 0.37 & 0.21 \\
\hline
\end{tabular}




\section{Factors associated with CRC Screening adherence}

Logistic regression analysis was used to test the combined influence of all variables on participation in screening (Table 4). Table 5 presents the final multiple logistic regression model for CRC screening adherence which was obtained after iterations. The participants who perceived fewer barriers (OR =.37; CI 95\%: .21- .89) more likely to utilize CRC screening. They who perceived more susceptibility (OR =2.99; CI 95\%: 1.23-5.45) were more likely carried out CRC screening. Regarding knowledge, the Participants with a high level of CRC knowledge were 1.29 times more likely to utilize CRC screening (OR $=1.29$; CI 95\%: 1.86-3.40).

\section{Discussion}

According to recommendations by WHO and American Association of Cancer Prevention, all individuals above 50 are subject to the risk of colorectal cancer, and they should perform fecal occult blood test every year and colonoscopy test every five years (Read and Kodner 1999). The study results showed that a high percentage of the participants have not information about CRC and CRC screening. Compared with a previous study conducted in Iran about knowledge toward CRC screening among people aged 50 years and over, the results showed that more than half of the participants had never heard about colorectal cancer screening tests (Salimzadeh et al., 2011). Omran et al (2010) in their study among 160 Jordanians, 50 years and older, reported low knowledge toward CRC screening (Omran and Ismail 2010) that is congruent with present study. Another study conducted in Iran about knowledge toward CRC screening among people aged 50 years and over, showed that more than half of the participants had never heard about CRC screening tests.

The results of our study demonstrated that the most common source of information were family members. There were high proportions of participants who were never informed about CRC screening by their doctor. In the most previous studies, physicians were as best practice guide for CRC screening tests (Powe et al., 2009; Ruffin IV et al., 2009). It could identify lack of doctor recommendation for CRC screening in our study.

In this study, perceived susceptibility to CRC had the lowest percentage of all of the subscales. Only $8 \%$ of the participants perceived that they were susceptible to contracting CRC, whereas $92 \%$ did not. In the study of Omran et al (2010), $41 \%$ of participants perceived that they were susceptible to contracting CRC.Also, a previous study conducted to investigate the frequency of CRC screening among African American women 50 years or older, showed that $50 \%$ of the participants did not perceive themselves susceptible to contracting CRC. However, perceived susceptibility to CRC should be caution because its ignorance caused that individuals did not participate in screening. These differences could be due to effect of cultural diversity on perceived susceptibility (Joseph et al 2012)

The findings also indicated more than half of participant's perceived benefits of CRC screening and believe that maintaining good health is extremely important to them. Participants had positive perceptions of early screening and agreed to seek and participate in screening. This result is congruent with a previous study conducted that examined predictors of breast and CRC screening behaviors among 600 women aged 50 to 75 years living in the south eastern United States, which showed the proportion of perceived benefits of CRC screening were high among participants.

In addition, this study revealed that CRC knowledge and two HBM-related factors, perceived susceptibility and barriers, were the major predictors of adherence to CRC screening. In African American women alone, Frank et al (2004) found that women who perceived greater susceptibility, benefits and self-efficacy had higher reports of screening compliance. Menon found that those in pre contemplation stage for CRC screening tests had lower perceived and higher barriers than both those in contemplation and those in action (Menon et al., 2007).

Few limitations to this study should be noted. Nonrandom sampling and limited sample size in the hospitals reduce generalizability of the findings and doing of study by a similar studies with larger sample sizes are suggested. The use of self-administered questionnaires might have led to information bias from participants who were unwilling to provide true information. Although the vast majority of studies in CRC screening are conducted on participants aged 50 or older, further research is recommended for other age groups and also high risk population since the Iranian inception age of CRC is lower than global average age.

In conclusion, our findings indicated lack of knowledge about CRC and CRC screening exists among Iranians. A high percentage of the participants did not perceive that they are susceptible to contracting CRC and more than half of participants perceived many barriers to screening. Our findings indicated that higher knowledge about CRC and perceived susceptibility and lower perceived barrier were good predictors of adherence to CRC screening. This implies that health professionals should put more focus on the increase of knowledge and two modifiable HBMrelated factors in order to encourage adults to adhere to CRC screening. Interventions such educational programs and motivational counseling strategies, which aim to lower barriers, and increase perception of susceptibility and CRC knowledge, should be developed and implemented as other studies showed (Bryan et al., 2015).

\section{Acknowledgements}

The researchers express their gratitude from Participants in this study as well as Research Deputy of Qom University of Medical Sciences to fund and support this project.

\section{References}

Alireza S, Mehdi N,Mohammad Ali M (2005). Cancer occurrence in Iran in 2002, an international perspective. Asian Pac J Cancer Prev, 6, 359.

Brennenstuhl S, Fuller-Thomson E, Popova S Prevalence and factors associated with colorectal cancer screening in 
Canadian women. $J$ Women's Health, 19, 775-784.

Bryan TJ, Estrada CA, Castiglioni A, et al (2015). impact of an educational intervention on provider knowledge, attitudes, and comfort level regarding counseling women ages 40-49 about breast cancer screening. J multidisciplinary healthcare, $\mathbf{8}, 209$.

Champion VL (1984). Instrument development for health belief model constructs. Advances in Nursing Science, 6, 73-85.

Champion VL (1999). Revised susceptibility, benefits, and barriers scale for mammography screening. Res Nurs Health, 22, 341-8.

Cole SR, Zajac I, Gregory T, et al (2011). Psychosocial variables associated with colorectal cancer screening in South Australia. International J Behavioral Medicine, 18, 302-9.

Ebu NI, Mupepi SC, Siakwa MP, et al (2015). Knowledge, practice, and barriers toward cervical cancer screening in Elmina, Southern Ghana. International J Women's Health, 7, 31 .

Gili M, Roca M, Ferrer V, et al (2006). Psychosocial factors associated with the adherence to a colorectal cancer screening program. Cancer Detection Prev, 30, 354-60.

Gimeno García AZ (2011). Factors influencing colorectal cancer screening participation. Gastroenterology research and practice, 2012.

Gonzalez P, Castaneda SF, Mills PJ, et al (2012). Determinants of breast, cervical and colorectal cancer screening adherence in Mexican-American women. J Community Health, 37, 421-33.

Haggar FA,Boushey RP(2009). Colorectal cancer epidemiology, incidence, mortality, survival, and risk factors. Clinics In Colon Rectal Surgery, 22, 191.

Henley SJ, King J, German RR, et al (2010). Surveillance of screening-detected cancers (colon and rectum, breast, and cervix), united states, 2004-2006, department of health and human services. Centers for Disease Control and Prevention.

Jacobs LA (2002). Health beliefs of first-degree relatives of individuals with colorectal cancer and participation in health maintenance visits, a population-based survey. Cancer Nurs, 25, 251-65.

Janz NK, Wren PA, Schottenfeld D, et al (2003). Colorectal cancer screening attitudes and behavior, a population-based study. Preventive medicine, 37, 627-634.

Javadzade SH, Reisi M, Mostafavi F, et al (2012). Factors associated with the fecal occult blood testing for colorectal cancer screening based on health belief model structures in moderate risk individuals, Isfahan, 2011. J Education and Health Promotion, 1.

Jorgensen ML, Young JM,Solomon MJ (2013). Adjuvant chemotherapy for colorectal cancer, age differences in factors influencing patients' treatment decisions. Patient Preference and Adherence, 7, 827.

Katz ML, Tatum C, Dickinson SL, et al (2007). Improving colorectal cancer screening by using community volunteers. Cancer, 110, 1602-10.

Kharameh ZT, Foroozanfar S, Zamanian H (2013). Psychometric properties of the persian version of champion's health belief model scale for colorectal cancer screening. Asian Pac J Cancer Prev, 15, 4595-9.

Mcfarland DM (2013). Associations of demographic variables and the Health Belief Model constructs with Pap smear screening among urban women in Botswana. International $J$ Women's Health, 5, 709.

Menon U, Belue R, Skinner CS, et al (2007). Perceptions of colon cancer screening by stage of screening test adoption. Cancer nursing, 30, 178.

Omran S,Ismail AA (2010). Knowledge and beliefs of Jordanians toward colorectal cancer screening. Cancer nursing, 33,
141-8.

Palmer RC,Emmons KM, Fletcher RH, et al (2007). Familial risk and colorectal cancer screening health beliefs and attitudes in an insured population. Preventive medicine, 45, 336-341.

Powe BD, Cooper DL, Harmond L, et al (2009). Comparing knowledge of colorectal and prostate cancer among African American and Hispanic men. Cancer nursing, 32, 412-7.

Read TE, Kodner I (1999). Colorectal cancer, risk factors and recommendations for early detection. American family physician, 59, 3083-92.

Ruffin Iv MT, Creswell JW, Jimbo M, et al (2009). Factors influencing choices for colorectal cancer screening among previously unscreened African and Caucasian Americans, findings from a triangulation mixed methods investigation. Journal of community health, 34, 79-89.

Salimzadeh H, Eftekhar H, Majdzadeh R, et al (2011). More than half of senior residents in tehran have never heard about colorectal cancer screening. Asian Pac J Cancer Prev, 12, 2851-6.

Sung JJ, Lau JY, Goh K, et al (2005). Increasing incidence of colorectal cancer in Asia, implications for screening. The lancet oncology, 6, 871-6.

Taylor VM, Schwartz SM, Jackson JC, et al (1999). Cervical cancer screening among Cambodian-American women. Cancer Epidemiology Biomarkers \& Prevention, 8, 541-6.

Ueland AS, Hornung PA,Greenwald B (2006). Colorectal cancer prevention and screening, a health belief model based research study to increase disease awareness. Gastroenterology Nursing, 29, 357-63.

Wang JH, Liang W, Chen MY, et al. (2006). The influence of culture and cancer worry on colon cancer screening among older Chinese-American women. Ethn Dis, 16, 404-11. 